drainage of her thoracic cavity was performed because of empyema at the time of referral. The left-chest anastomosis appeared well drained by means of computed tomographic analysis, and contrast study showed no evidence of ongoing leak and a well-positioned stent. ${ }^{2}$ The patient was followed closely in the hospital and maintained on intravenous antibiotics. Nutritional support was through a jejunostomy feeding tube. The esophageal stent was removed at 4 weeks after esophagectomy and replaced because the defect appeared to be healing well. The patient continued her slow improvement, and then at 6 weeks after esophagectomy, she had abrupt onset of massive gastrointestinal bleeding and hematemesis with hemodynamic compromise. ${ }^{3}$ She underwent esophagogastroduodenoscopy in the operating room, which demonstrated a gastroaortic fistula on removal of the stent. An emergency thoracotomy was performed with primary repair of the aorta and esophageal diversion with cervical esophagostomy. ${ }^{4}$ She had a difficult postoperative course and died 2 weeks later from multisystem organ failure.

\section{DISCUSSION}

Our group has had an extensive experience with esophageal stenting for leaks and perforations. This patient came to us septic and debilitated from an uncontrolled postesophagectomy leak that had been present for about 3 weeks. As part of our standard approach, we placed an esophageal stent to exclude the leak and then drained the active infection by means of thoracoscopy. This is the only patient we have treated using this algorithm who has had a left thoracoabdominal approach for resection. The proximity of the aorta and resultant infection are clearly concerns for this approach in retrospect. Although the stent was positioned well and the patient was recovering acceptably from her septic insult, the residual infection around the aorta and the spatial relationship of the stent to the aorta led to clear erosion of the distal aspect of the stent through the proximal stomach and into the aorta.

In the patient with a leak who is seen after esophagectomy, the complication of gastroaortic fistula has not been reported previously. This case represents one of the first reports of this complication related to the use of esophageal stents ${ }^{5}$ and should raise concerns regarding esophageal stenting for leak after a left thoracoabdominal approach for esophagectomy because of anatomic considerations. ${ }^{4}$ There is little doubt that the periaortic infection from the leak contributed substantially to the eventual development of the gastroaortic fistula. As esophageal stents become used more widely and with increasing frequency, one should be cautioned regarding this potential complication.

\section{References}

1. Freeman RK, Ascioti AJ, Wozniak TC. Postoperative leak management with the Polyflex esophageal stent. J Thorac Cardiovascular Surg. 2007;133:333-8.

2. Korst RJ, Port JL, Lee PC, Altorki NK. Intrathoracic manifestations of cervical anastomotic leaks after transthoracic esophagectomy for carcinoma. Ann Thorac Surg. 2005;80:1185-90.

3. Tak AM, Klizek RD, Campion JP. Primary aorto-esophageal fistula: a rare cause of gastrointestinal hemorrhage. Internet Journal of Internal Medicine. 2005. Available at: http://www.ispub.com/.../primary_aorto_esophageal_fistula_a_rare_ cause_of_gastrointestinal_hemorrhage.html. Accessed March 16, 2009.

4. Unosawa S, Hata M, Sezai A, Niino T, Yoda M, Shimura K, et al. Surgical treatment of an aortoesophageal fistula caused by stent implantation for esophageal stenosis: report of a case. Surg Today. 2008;38:62-4.

5. Chandrashekar G, Kumar VM, Kumar AK. Repair of aortoesophageal fistula due to a penetrating atherosclerotic ulcer of the descending thoracic aorta and literature review. J Cardiothorac Surg. 2007;2:12.

\title{
Giant mycotic pseudoaneurysm of the left main coronary artery after pneumococcal pneumonia
}

\author{
Dimitri Kalavrouziotis, MD, and François Dagenais, MD, Sainte-Foy, Québec, Canada
}

\footnotetext{
From the Department of Cardiac Surgery, Institut Universitaire de Cardiologie et de Pneumologie, Sainte-Foy, Québec, Canada.

Disclosures: None.

Received for publication Nov 14, 2009; accepted for publication Nov 22, 2009; available ahead of print March 17, 2010.

Address for reprints: Francois Dagenais, MD, Laval Hospital, Department of Cardiac Surgery, 2725 chemin Sainte-Foy, Québec, Québec G1V 4G5, Canada (E-mail: francois.dagenais@chg.ulaval.ca).

J Thorac Cardiovasc Surg 2010;140:e50-2 $0022-5223 / \$ 36.00$

Copyright $\Subset 2010$ Published by Elsevier Inc. on behalf of The American Association for Thoracic Surgery

doi:10.1016/j.jtcvs.2009.11.054
}

Coronary artery aneurysms are mainly atherosclerotic in origin. ${ }^{1}$ Conversely, mycotic coronary artery aneurysms are extremely uncommon. We describe a unique case of mycotic pseudoaneurysm of the left main coronary artery after pneumococcal pneumonia.

\section{CLINICAL SUMMARY}

A 62-year-old man without a history of cardiac disease had chest discomfort of 3 weeks' duration. The chest pain was exertional but also varied with the patient's position. 


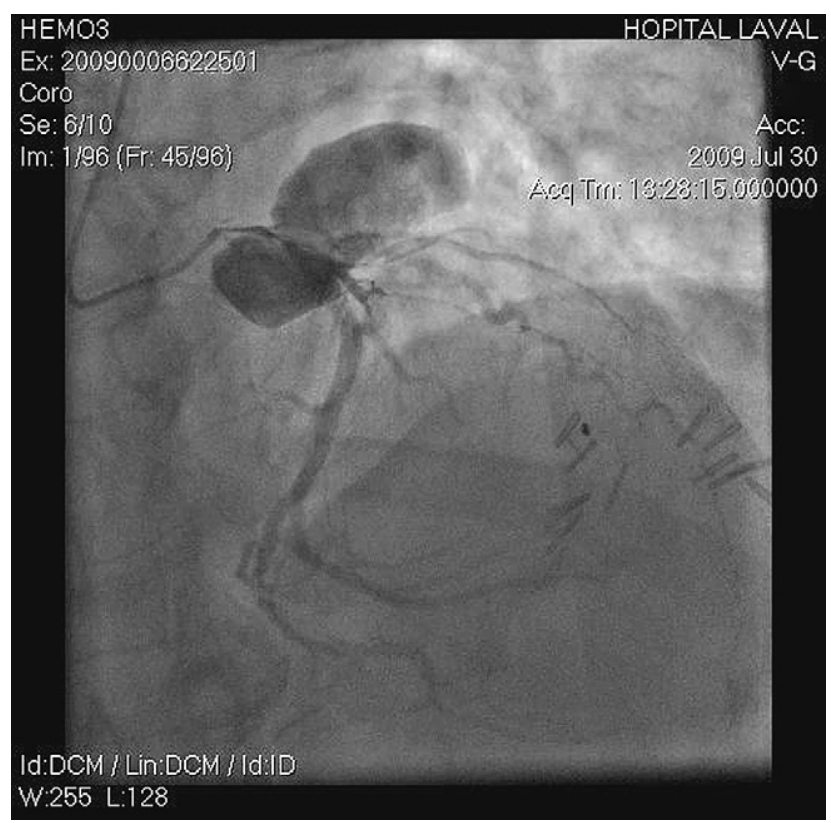

FIGURE 1. Left coronary arteriography showing a contrast-filled space in association with the left main coronary artery.

The patient's history was notable for chronic lymphocytic leukemia that had necessitated a splenectomy. Three months before presentation, he had had pneumococcal pneumonia complicated by septic arthritis and a pericardial effusion. A pericardial window was performed at an outside hospital and cultures were positive for Streptococcus pneumoniae. A full course of appropriate intravenous antibiotic therapy was completed with a satisfactory clinical response.

On arrival, an exercise stress test was clinically and electrically positive at moderate workload and was associated with ventricular ectopy and hypotension. Coronary angiography showed a multilobulated contrast-filled space in association with the left main coronary artery (Figure 1). The left anterior descending artery (LAD) was occluded but collateralized and a significant stenosis was present in the proximal circumflex artery, which was dominant. Echocardiography demonstrated a moderate hypokinesis of the anterolateral and apical walls with an ejection fraction of $40 \%$ without signs of endocarditis. Chest computed tomography (CT) revealed a 6-cm pseudoaneurysm bulging behind and lateral to the main pulmonary artery. The origin was thought to be the left main coronary artery without communication with the aortic root (Figure 2). There was no evidence of aneurysm on a previous CT performed 3 months earlier for the evaluation of effusive pericarditis. On clinical assessment, the patient appeared well and was in hemodynamically stable condition. Oral temperature was $38.2^{\circ} \mathrm{C}$ and peripheral blood cultures were negative.

The patient was taken to the operating room on an urgent basis. Dense adhesions were encountered owing to the previous pericarditis. After cardiopulmonary bypass and aortic crossclamping, the main pulmonary artery was divided to facilitate exposure of the aneurysm. The aneurysm was entered and its thickened and inflammatory walls were excised. An aortotomy excluded the presence of a fistulous tract originating from the aortic root. The proximal left main coronary artery was ligated. A saphenous vein was interposed between the aortic root and the proximal circumflex artery in the atrioventricular groove. The LAD and posterior descending arteries were grafted with the left internal thoracic artery and a saphenous vein, respectively. A segment of pulmonary homograft was interposed to avoid undue tension on the pulmonary artery reconstruction. Intraoperative cultures were negative.

Postoperative recovery was largely uneventful except for a mild self-limited delirium. The patient was discharged on a 3-week oral antibiotic course. At 3 months' follow-up, the patient was well and without complications. A chest CT demonstrated patent grafts without recurrent pseudoaneurysm.

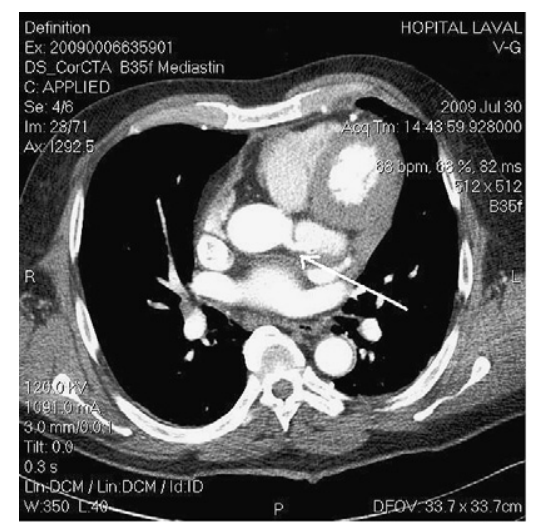

A

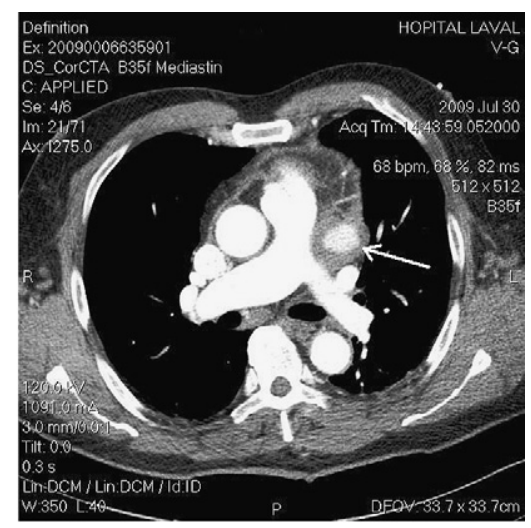

B

FIGURE 2. Contrast-enhanced computed tomographic scan of the chest. A, A saccular aneurysm of the left main coronary artery is seen. The left main coronary artery is shown by an arrow. B, The aneurysm (arrow) extends cephalad along the left lateral surface of the main pulmonary artery. 


\section{DISCUSSION}

Mycotic aneurysms of the coronary arteries are exceedingly uncommon, accounting for less than $3 \%$ of all coronary aneurysms. ${ }^{2}$ Most are associated with infective endocarditis, although coronary stents are increasingly implicated in the formation of mycotic coronary aneurysms. ${ }^{2,3}$ We present a unique case of a mycotic aneurysm of the left main coronary artery in a splenectomized patient after a pneumococcal pneumonia complicated by purulent pericarditis. The rapid growth of the pseudoaneurysm within 3 months coupled with the septicemia and purulent pericarditis support the mycotic origin of the pseudoaneurysm. Pre-existing coronary calcifications may have led to an initial endarteritis followed by a contained rupture and purulent pericarditis. Although the presentation was ischemic in nature owing to coronary artery stenosis, it was likely that the pseudoaneurysm caused compression on the coronary arteries as suggested by the positional feature of the patient's symptoms.

Preoperative assessment is essential to define the relationship of the pseudoaneurysm to the surrounding structures. High-resolution multislice CT allows excellent visualization of the proximal coronary arteries and relationships with surrounding structures. Although covered stents have been reported as a treatment for mycotic coronary pseudoaneurysm, open surgical management remains the gold standard for patients without prohibitive risk. ${ }^{4,5}$ Access to a pseudoaneurysm of the left main coronary artery requires transection of the main pulmonary artery in most circumstances, especially in the presence of dense adhesions, as in the present case. Unroofing of the aneurysm wall with proximal ligation of the coronary artery is mandatory. Although technically challenging, in situ revascularization of the proximal circumflex artery is feasible, as performed in our case. The proximal LAD is often difficult to revascularize and is probably best revascularized with the left internal thoracic artery. Appropriate antibiotic treatment is essential to minimize recurrence.

\section{References}

1. Nichols L, Lagana S, Parwani A. Coronary artery aneurysm: a review and hypothesis regarding etiology. Arch Pathol Lab Med. 2008;132:823-8.

2. Garg N, Garg R, Gordon C, Singh R, Singh A. Acute coronary syndrome caused by coronary artery mycotic aneurysm due to late stent infection localized with radiolabeled autologous leukocyte imaging. Clin Nucl Med. 2009;34:753-5.

3. Schoenkerman AB, Lundstrom RJ. Coronary stent infections: a case series. Catheter Cardiovasc Interv. 2009;73:74-6.

4. Christiansen S, Klocke A, Hoffman A, Autschbach R. Surgical management of a left main stem coronary artery aneurysm. J Card Surg. 2008;23:69-70.

5. Shariff N, Combs W, Roberts J. Large mycotic pseudoaneurysm of the left circumflex treated with antibiotics and covered stent. J Invasive Cardiol. 2009;21:E37-8.

\title{
Pleural pressure immediately after pulmonary lobectomy: Single versus double chest tubes for suction
}

\author{
Alessandro Brunelli, MD, ${ }^{\mathrm{a}}$ Stephen D. Cassivi, MD, ${ }^{\mathrm{b}}$ Juan Fibla, MD, ${ }^{\mathrm{b}}$ and Luca Di Nunzio, MD, ${ }^{\mathrm{a}}$ \\ Ancona, Italy, and Rochester, Minn
}

Recent studies have shown that a single chest tube after pulmonary lobectomy is at least as effective as 2 chest tubes in evacuating air and fluid from the pleural space. ${ }^{1-3}$ In addition, a single tube may produce less pain and may be a more suitable option for patients being treated with fast-tracking approaches. Most surgeons still favor the use of $-20 \mathrm{~cm}$

\footnotetext{
From the Division of Thoracic Surgery, ${ }^{\text {a }}$ Ospedali Riuniti, Ancona, Italy, and the Division of General Thoracic Surgery, ${ }^{\mathrm{b}}$ Mayo Clinic, Rochester, Minn.

Disclosures: Dr Brunelli is a member of the International Advisory Board of Medela AG, Baar, Switzerland.

Dr Fibla is the recipient of the AATS Evarts Graham Traveling Fellowship 2009-2010.

Received for publication Feb 1, 2010; accepted for publication April 10, 2010; available ahead of print May 14, 2010.

Address for reprints: Alessandro Brunelli, Division of Thoracic Surgery, Ospedali Riuniti Ancona, Via Conca 71, 60020, Ancona, Italy (E-mail: brunellialex@ gmail.com).

J Thorac Cardiovasc Surg 2010;140:e52-3

$0022-5223 / \$ 36.00$

Copyright (c) 2010 by The American Association for Thoracic Surgery doi: $10.1016 /$ j.jtcvs. 2010.04 .013
}

$\mathrm{H}_{2} \mathrm{O}$ suction in the first postoperative hours, with the theoretic aim of promoting lung expansion. This level of suction has persisted in most practices since being adapted from early thoracic drainage devices designed by Emerson in the 1940s. The benefits of chest tube suction, however, remain a topic of debate. This study investigated whether the use of 1 or 2 chest tubes after pulmonary lobectomy might influence the level of intrapleural pressure as measured in the first postoperative hours.

\section{MATERIALS AND METHODS}

The study was approved by the local hospital institutional review boards, and patients gave informed consent for collection and use of their data in the database for research purposes. This post hoc analysis of a prospective study to assess the efficacy of a novel chest drain system included 145 patients undergoing pulmonary lobectomy through a lateral muscle-sparing thoracotomy in 2 separate thoracic surgery centers (2007-2008). After completion of the lobectomy, 2 chest tubes ( 1 at the apex and the other placed at the base) or 1 chest tube (placed in a lateral position up to the apex, all tubes 